\title{
Metodología para el pronóstico de la demanda en ambientes multiproducto y de alta variabilidad
}

\section{Methodology to demand forecastingunder multiproduct environments and high variability}

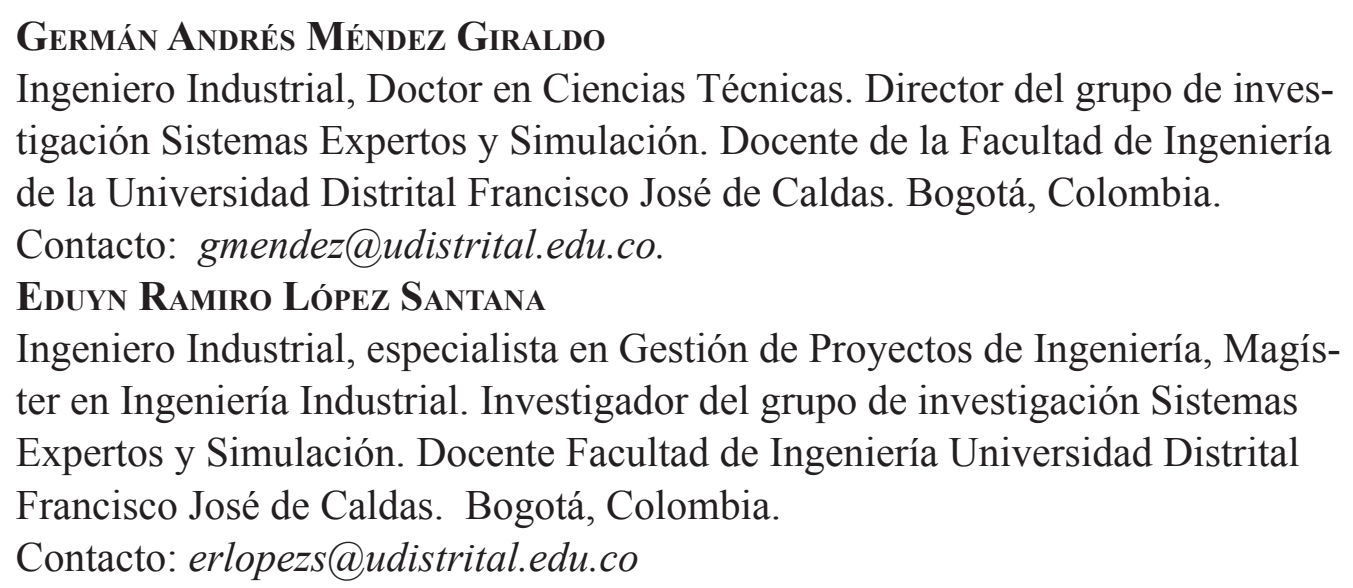

Palabras clave: clasificación abc, planeación de producción, pronóstico de la demanda, sistemas de tiempo variable.

Key words: ABC Classification, Demand Forecasting, Production Planning, Time Varying Systems.

\section{RESUMEN}

Este artículo presenta una metodología para la gestión de la demanda en ambientes multiproducto para diferentes eslabones de la cadena de suministro y con alta variabilidad en la demanda. La metodología propuesta consiste en dos fases: la primera busca una clasificación de los productos de acuerdo con dos criterios, uno de movimiento para representar la rotación de inventarios, y el segundo de importancia respecto a las variables costo, volumen y peso; el resultado de esta fase es un conjunto de categorías para clasificar los productos e identificar aquellos más importantes y aquellos que se deben descontinuar. La segunda fase consiste en realizar un pronóstico agregado de acuerdo con la variabilidad que se presenta en cada una de las categorías identificadas, se emplea el coeficiente de variación como medida de varia- 
bilidad y suavización exponencial doble y promedios móviles dobles como técnica de pronóstico, aunque la metodología es flexible y se pueden emplear otros métodos de series de tiempo. La metodología se implementó en una empresa líder en Colombia de productos polivinilos logrando una mejora del $10 \%$ en la desviación absoluta promedio, aproximadamente. Por otro lado, se logró una reducción significativa en el inventario promedio de todo el sistema. Finalmente, este trabajo es una fase inicial para una clasificación dinámica de los productos para gestionar de forma integral los inventarios en una cadena de múltiples eslabones.

\section{ABSTRACT}

This paper provides a methodology for the demand management in multiproduct environments for several stages in a supply chain with high variability in the demand. The methodology proposed consists of two phases. The first phase looks for products classification according to two criterions, the first is the movement factor representing inventory's turns and the second is the importance factor given by variables such as cost, volume and weight. The output of this phase is a product category to identify the most important products and which should be discontinued. The second phase consists in performing an aggregate forecast according to the variability in each category. We use the coefficient of variation (CV) as measure of variability, and the double exponential smoothing method and the double moving average method as forecasting techniques, although the methodology is flexible to use another time series methods. The methodology was implemented in a leader company in Colombia of poly vinyl products achieving an improve of $10 \%$ in the mean absolute deviation, approximately. On the other hand the average inventory in the system was reducing significantly. Finally this work is a first step for a dynamic classification of goods in order to manage inventory holistically a multistage supply chain.

\section{INTRODUCCIÓN}

En la actualidad, las empresas buscan la mejora en los procesos de todas las áreas de su estructura organizacional, utilizando de una u otra forma los conceptos y las herramientas de la gestión de procesos; sin embargo, en el presente se reconoce la importancia de disponer de una metodología de integración, donde cobra especial atención las cadenas de suministro. En estos procesos de mejora se requiere el correcto manejo de los procesos críticos de estimación de la demanda, la planeación de inventarios, la planeación de los recursos de producción, distribución y la programación de la producción y planeación de materiales. De estos cuatro procesos se considera que se debe iniciar por un buen método de estimación de la demanda, como decisión estratégica fundamental para una organización, que es la base inicial para

la toma de decisiones. La metodología desarrollada se basa en los modelos de gestión integral para sistemas donde existe un conjunto de familias, tipos, clases, entre otros, que son considerados como sistemas multiproducto y que se basan en una cadena de abastecimiento que recoge las necesidades de los mayoristas y minoristas, por lo que se considera multieslabon, se propone una estrategia que coayude a aumentar la productividad y servicio de la organización, ya que tradicionalmente las aplicaciones tipo ERP (Enterprise Resource Planning) manejan los pronósticos en una estructura abajo hacia arriba faltando aún el proceso de doble vía, es decir de arriba hacia abajo. Se requiere que los pronósticos sean para distribuidores, centro de distribución, bodega y por planta. Esta metodología surge como resultado de un proyecto avalado y financiado por una empresa líder en Colombia del sector de tuberías 
y desarrollado por el grupo de investigación Sistemas Expertos y Simulación (SES) adscrito a la Facultad de Ingeniería de la Universidad Distrital Francisco José de Caldas.

Este artículo está organizado en seis partes incluyendo esta introducción. En el segundo apartado se muestra el marco referencial donde se abarcan generalidades sobre la clasificación de inventarios y de técnicas de pronósticos. En la tercera parte se describe el caso de aplicación, seguido en el apartado cuarto se encuentra la metodología propuesta con los principales resultados encontrados. En el apartado quinto se presentan las principales conclusiones del trabajo, para finalizar con unos comentarios sobre trabajos futuros en el sexto apartado y las referencias bibliográficas.

\section{MARCO REFERENCIAL}

\section{Revisión de la literatura}

Respecto a la clasificación de inventarios y productos se han realizado diferentes trabajos como en el que utilizan una aproximación de modelación matemática para establecer las diferentes agrupaciones de productos utilizando una metodología ABC. Como en Chu, Liang y Liao (2008) donde se emplea una metodología basada en conjuntos difusos para la clasificación y control de inventario llamado $\mathrm{ABC}$-fuzzy clasificación ( $\mathrm{ABC}-\mathrm{FC})$, que puede manejar las variables lingüísticas que incorpora la experiencia del gerente al juicio en la clasificación del inventario, y se pueden implementar fácilmente, encontrando una alta precisión de clasificación. Por otro lado, en Chen (2011) se utiliza un enfoque donde se determinan dos tipos comunes de pesos y se agregan múltiples criterios de manera objetiva, de forma que se logre un indicador de desempeño más racional en comparación con las metodologías clásicas. En Torabi, Hatefi y Saleck Pay (2012) se presenta un sistema de apoyo a las decisiones para el análisis y el control de inventarios mediante tres diferentes tipos de análisis basado en: precio, cantidad e importan- cia $(\mathrm{ABC})$, mostrando una aplicación del método al inventario de planta de gas, buscando aislar las partes más críticas en términos de sus precios y cantidades respectivamente.

También se pueden utilizar metodologías basadas en múltiples criterios como las mostradas en Hadi-Vencheh (2010), o metodologías subjetivas como en $\mathrm{Ng}$ (2007), así mismo, algunas basadas en métodos de optimización con múltiples criterios dando ponderaciones a estos, como en Ramanathan (2006). La utilización de métodos de optimización para la clasificación de inventarios también ha incursionado en la aplicación de técnicas de inteligencia artificial, como algoritmos genéticos, redes neuronales, sistemas de decisión basados en reglas como los mostrados en Partovi y Anandarajan (2002); Rezaei y Dowlatshahi (2010); Tsai y Yeh (2008).

\section{Clasificación del inventario}

De acuerdo con Arora (2005), el inventario puede ser clasificado en los aspectos de manufactura, servicio y control. Así mismo, en el primer caso, se tienen los inventarios de materia prima, componentes y subensambles comprados a terceros; que se almacenan con el fin de asegurar su disponibilidad para la producción (Askin y Goldberg, 2002). En este caso, se debe considerar la proximidad del proveedor, relación con el proveedor, predecibilidad del proceso de producción, lead time requerido para poner una orden, y transportabilidad y perecibilidad de los materiales. También se tienen los inventarios de producto en proceso (WIP, Work In Process) o artículos en forma semiterminada o con diferentes etapas de producción; en este caso, se manejan lotes de material y partes compradas, desde el momento en que son liberadas al taller hasta que hacen parte del producto terminado (Askin y Goldberg, 2002). Otro grupo son los inventarios de producto terminado, incluye los productos listos para despachar a los clientes (Hopp y Spearman, 2008). Se consideran 
los inventarios de tubería - pipeline- que son los productos en tránsito entre instalaciones - fábricas-, que incluye materia prima siendo entregada a la planta, y productos finales siendo enviados a las bodegas o a los clientes (Askin y Goldberg, 2002), igualmente, cuando el movimiento es lento, entre grandes distancias o debe tener lugar entre muchas etapas, la cantidad de inventario en la tubería podría incluso exceder la cantidad mantenida en los puntos de almacenaje (Ballou, 2004). Un grupo importante es el de los inventarios MRO que incluyen provisiones de mantenimiento, reparación y operación como partes separadas y material consumible, los cuales no van al producto final pero son consumidos en el proceso de producción.

Cuando el control de inventarios abarca cientos o miles de ítems, crea un problema serio para la administración, llevar registro de cada uno de los ítems y tener la misma capacidad de control en cada uno de ellos. En general, se puede decir que el exceso y la escasez no afectan a todos los ítems en almacenamiento, entonces es mucho más fácil dividir los ítems en un número de grupos —agruparlos- y utilizar las mismas técnicas de pronósticos y control de inventarios para todos los ítems de un mismo grupo. La agrupación de los artículos se puede hacer en muchas maneras diferentes y debe depender de las necesidades de la organización (Ballou, 2004), por ejemplo, el inventario de una organización industrial consiste generalmente de miles de ítems con diferentes precios, tasas de consumo y lead times, para los cuales no es deseable ni posible prestar igual atención a todos los ítems. El análisis ABC (Always Better Control) es una herramienta analítica básica que permite a la administración concentrar sus esfuerzos en donde éstos tendrán un mayor efecto (Hopp y Spearman, 2008), las estadísticas revelan que solo pocos ítems contribuyen para la mayoría del consumo anual de dinero, es decir, se comportan de acuerdo con el principio de Pareto de pocos vitales y muchos triviales. Estos pocos ítems son llamados ítems de clase A, los cuales son claves para el negocio, ya que su existencia constituye la mayoría de inversión en recursos de inventario, los otros ítems conocidos como B y C son numerosos en cantidad pero su contribución es menos significativa.

Es común usar estas tres clasificaciones de prioridad: A (más importantes), B (de importancia intermedia) y $\mathrm{C}$ (menos importantes). El número de categorías apropiado para una compañía depende de sus circunstancias — tamaño de la empresa, tamaño del inventario, número de ítems controlados, entre otros- y el grado al que se desee diferenciar la cantidad de esfuerzo asignado a las agrupaciones. Aplicar la mayoría de los recursos limitados de planeación y control a los A-ítems -mayor rendimiento económico-, mientras que los gastos en las otras clases que tienen mucho menos efecto sobre la inversión total del inventario son mantenidos en un mínimo. De esta forma, el esfuerzo y los recursos ahorrados a través de la relajación del control en ítems de bajo valor (ítems B y C) serán destinados a reducir inventarios de ítems de alto valor (Ítems A).

\section{Técnicas de pronósticos}

Pronosticar es la ciencia y arte de predecir eventos futuros (Méndez Giraldo, 2003). Puede implicar tomar datos históricos y proyectarlos hacia el futuro con alguna clase de modelo matemático, también puede ser una predicción subjetiva o intuitiva, o puede implicar una combinación de estas, es decir, un modelo matemático ajustado por el buen juicio de un administrador. Raramente existe una técnica superior a las otras, lo que trabaja mejor en una compañía bajo un conjunto de condiciones puede ser un completo desastre en otra organización, o aún en un departamento diferente de la misma firma. Los pronósticos son rara vez perfectos, son costosos y toman tiempo considerable para prepararse y para ser monitoreados (Méndez Giraldo, 2003). Sin embargo, debido a que la planeación estratégica efectiva, tanto en el corto como en el largo plazo, depende de un pronóstico de la demanda de los productos de la 
compañía, es inevitable enfrentar la necesidad de hacer pronósticos (Heizer y Render, 2010).

Este pronóstico tiene una longitud temporal dependiente del tipo de daciones que se deban establecer, según el enfoque Jerárquico existirán pronósticos de largo plazo, mediano plazo y corto plazo, correspondientes a daciones de tipo estratégico, táctico y operativo, respectivamente. Los pronósticos de corto plazo son necesarios para prácticamente todos los departamentos de una organización, sin embargo, se usan más frecuentemente en las áreas de mercadotecnia, producción y administración de la cadena de suministro, empleando usualmente diferentes metodologías que los de mayor plazo. Las técnicas matemáticas, como promedios móviles, suavización exponencial y extrapolación de tendencia, son comunes en las proyecciones de corto plazo (Heizer y Render, 2010).

Adicionalmente, los pronósticos de corto plazo tienden a ser más precisos que los de largo plazo (Méndez Giraldo, 2003). Los factores que influencian la demanda cambian día a día, de esta manera, en la medida en que el horizonte de tiempo se alarga es probable que la precisión del pronóstico disminuya. Por esta razón, los pronósticos de la demanda deben actualizarse regularmente con el fin de mantener su valor e integridad. Después de cada periodo de ventas, deben revisarse los pronósticos (Méndez Giraldo, 2003). Para construir un sistema de pronósticos se necesitan por lo menos ciertos datos iníciales, una técnica de pronósticos y los métodos de revisión y monitoreo necesarios para actualizar el modelo.

Se han desarrollado varias técnicas de pronósticos que se pueden clasificar principalmente en dos categorías: métodos cuantitativos y métodos cualitativos (Borisov, Bulanov, Orlova y Kondratov, 2008). Los primeros se usan cuando se tiene disponible información cuantitativa suficiente. Estos a su vez se pueden clasificar en: series de tiempo (extrapolativos) que predicen la continuación de patrones históricos y los explicativos (causales o estructurales) que pretenden ayudar a entender cómo las variables explicativas, como por ejemplo el precio, afectan la demanda. Los segundos se utilizan cuando la información no es disponible, es un producto nuevo, entre otras, empleando técnicas como el método Delphi, curvas de aprendizaje, curvas logísticas, investigación de mercados, entre otras (Borisov et al., 2008; Heizer y Render, 2010).

\section{CASO DE APLICACIÓN}

Para la implementación y validación de la metodología se tomó una empresa líder en Colombia del sector industrial en la fabricación de tuberías y productos polivinilos, con una cobertura del mercado nacional e internacional, comparando el desempeño y confiabilidad de la información durante un período de seis meses, utilizando en paralelo esta metodología con el uso de las facilidades brindadas en una aplicación del tipo ERP. La empresa cuenta con un portafolio de productos superior a $3700 \mathrm{~S}$. K. U, con tres plantas de producción, 14 centros de distribución en el ámbito nacional y una clientela que se aproxima a los 3500 clientes entre públicos y privados. El sistema en la actualidad tiene capacidad disponible para la producción, sin embargo tiene una ocupación total dejando demanda insatisfecha, esto quiere decir que el cuello de botella es la capacidad de las plantas y no el mercado, entonces, los pronósticos deben servir para regular la utilización de las capacidades de las distintas plantas y mejorar la respuesta al cliente interno y externo. La efectividad del pronóstico es cercana al $66 \%$ de forma agregada, pero desde luego la meta es incrementarla, permitiendo un proceso de estimación flexible que facilite el análisis de información útil al proceso de toma de decisiones.

En general se tiene un cubrimiento de existencias que permiten cumplir las demandas de 30 días y la meta es alcanzar un cubrimiento de 20 días, pasando por un objetivo intermedio de cubrimiento igual a 25 días. Esto indica que se debe disminuir 


\section{reflexión}

el número de existencias - tamaño del inventario- sin afectar el nivel de servicio que se estima cualitativamente en un nivel del $80 \%$. Se han clasificado los inventarios de producto final a través de cuadrantes, en donde el $100 \%$ de productos se analizan por las variables volumen de los tamaños de los pedidos y la frecuencia en que se requieren. Esta estructura indica que Q1 es el cuadrante en donde se ubican los productos de tamaño de pedido alto y gran frecuencia en su solicitud denominado ítems para mantener en existencia, mientras que en Q4 se ubican los de alto tamaño y poca frecuencia y son producidos.

\section{METODOLOGÍA PROPUESTA}

La metodología propuesta busca integrar la clasificación de productos - se utilizará la palabra producto e ítem indistintamente- con las técnicas de pronóstico, para establecer un módulo de gestión de demanda para una organización. A continuación se describen las generalidades de las dos partes principales de la metodología.

\section{Módulo de clasificación de productos}

Para realizar el proceso de clasificación se tomaron varios criterios globales: el primero, por la cantidad de movimiento que ha presentado el ítem, garantizando de esta manera suavizar los efectos de la alta variabilidad de la demanda, este es denominado por movimiento. El segundo es un criterio de importancia, el cual se considera como un criterio compuesto.

\section{CLASIFICACIÓN POR MOVIMIENTO}

El criterio por movimiento se basa en las estadísticas de los últimos dos años. Se compone de seis clases de la siguiente manera (ver tabla 1):

- Clase 1: ítems con el total de observaciones, esto es con 24 datos.

- Clase 2: ítems con hasta 21 datos de movimiento.

- Clase 3: ítems que tienen menos de 12 datos pero con movimiento en último semestre.

- Clase 4: ítems que no han tenido movimiento en el último semestre.

- Clase 5: ítems que no han tenido movimiento en el último año.

- Clase 6: ítems que no han tenido movimiento en los últimos dos años.

Tabla 1. Criterios de clasificación según movimiento

\begin{tabular}{|c|c|c|c|c|}
\hline Clase & Rango & Acción & \%Part. & SKU \\
\hline Clase 1 & Más de 21 movimientos & Análisis completo & 35,9 & 1109 \\
\hline Clase 2 & Menos de 21 movimientos & Análisis completo & 21,9 & 678 \\
\hline Clase 3 & $\begin{array}{c}\text { Movimiento con menos de 12 datos pero con } \\
\text { movimiento en último semestre }\end{array}$ & Mirar 9 meses más & 18,1 & 559 \\
\hline Clase 4 & Movimiento con 0 valores Último Semestre & Mirar 6 meses más & 9,4 & 291 \\
\hline Clase 5 & Movimiento <=12 y 0 en Último Año & Dar de baja Prioridad 2 & 12,3 & 381 \\
\hline Clase 6 & Movimiento con 0 valores & Dar de baja Prioridad 1 & 2,3 & 71 \\
\hline & & TOTAL & 100,0 & 3089 \\
\hline
\end{tabular}

Fuente: elaboración propia. 
De las seis clases de movimiento se eliminan las clase 5 y 6 dado que, por un lado, no se pueden analizar para la estimación de la demanda y, por el otro, se debe establecer un procedimiento para dar de baja a este tipo de productos -, es decir vender o dar salida de inventario físico-

\section{Clasificación por importancia}

Para la construcción del criterio de importancia se tomaron tres variables, la primera de carácter económico y las otras dos de carácter físico, con esto se protege a la organización de racionalizar sus espacios de bodegaje. La primera toma el costo, para las variables físicas se toma el peso y el volumen. Estas tres variables fueron modificadas por el valor de ventas (demanda total) de los datos históricos suministrados. Creando cuatro categorías $\mathrm{A}, \mathrm{B}, \mathrm{C}$ y D, y afectadas por un porcentaje de composición en el cual el costo lleva un peso de $70 \%$ de la calificación y los otros dos criterios, peso y volumen, un $30 \%$ del peso relativo. En la tabla 2 se dan los porcentajes de calificación.

Tabla 2. Clase para criterios de importancia

\begin{tabular}{|c|c|}
\hline Clase & Porcentaje participación \\
\hline A & $<=70$ \\
\hline B & $<=90$ \\
\hline C & $<=95$ \\
\hline D & $<=100$ \\
\hline
\end{tabular}

Fuente: elaboración propia.

Se clasifica teniendo en cuenta los criterios de movimiento y de importancia, en principio se tienen 18 categorías tal y como se muestra en la figura 1, como se mencionó en el caso de ejemplificación, se tuvieron que eliminar las clases 5 y 6 por no contar con demanda en los últimos meses. Para esta nueva reclasificación también se debe observar la cantidad de valores que compone cada clase y, como se puede observar, los valores de E2-C, E3-A, E3-B, E3-C, E4-A, E4-B y E4-C son pequeñas muestras es decir con menos de 30 observaciones con lo cual se puede dificultar su análisis.

\begin{tabular}{|c|c|c|c|c|c|c|c|}
\hline & & & & ovm & & & \\
\hline & & E1 & $E_{2}$ & E3 & E4 & ES & E6 \\
\hline & & 31 & 11 & 0 & 1 & 241 & 37 \\
\hline$u_{\underline{z}}$ & B & 39 & 16 & & & 113 & 19 \\
\hline$\overline{\underline{\underline{z}}}$ & $\mathrm{c}$ & 32 & 9 & 7 & 9 & 27 & 15 \\
\hline & D & 1007 & 642 & 547 & 278 & & - \\
\hline
\end{tabular}

Figura 1. Clasificación de los ítems de inventario

Fuente: elaboración propia.

Por ello se decide trabajar con dos reclasificaciones en importancia Clase A y Clase B, con valores de participación en la importancia hasta el 95\% acumulado para la clase A y del 100\% acumulado para la clase B, respectivamente. En general, se puede decir que la nueva clase $\mathrm{A}$ es la integración de la Clase A, B y C, mientras que la nueva clase $\mathrm{B}$ es la clase D. En la figura 2 se muestra el flujograma de la metodología de clasificación de ítems propuesta.

\section{Módulo de estimación de la demanda}

Para realizar el proceso de pronosticar los productos, se toma una muestra de datos para las clases 1 a 4, los valores se muestran en la tabla 3 .

Tabla 3. Cantidad de elementos a pronosticar

\begin{tabular}{|c|c|c|}
\hline \multicolumn{3}{|c|}{ PRODUCTOS } \\
\hline \multirow{2}{*}{ Clase } & Tipo & Ítems seleccionados \\
\hline \multirow{2}{*}{1} & A & 20 \\
\cline { 2 - 3 } & B & 30 \\
\hline \multirow{2}{*}{2} & A & 7 \\
\cline { 2 - 3 } & B & 19 \\
\hline \multirow{2}{*}{3} & A & 3 \\
\cline { 2 - 3 } & B & 16 \\
\hline \multirow{2}{*}{4} & A & 3 \\
\cline { 2 - 3 } & B & 8 \\
\hline
\end{tabular}

Fuente: elaboración propia. 


\section{reflexión}

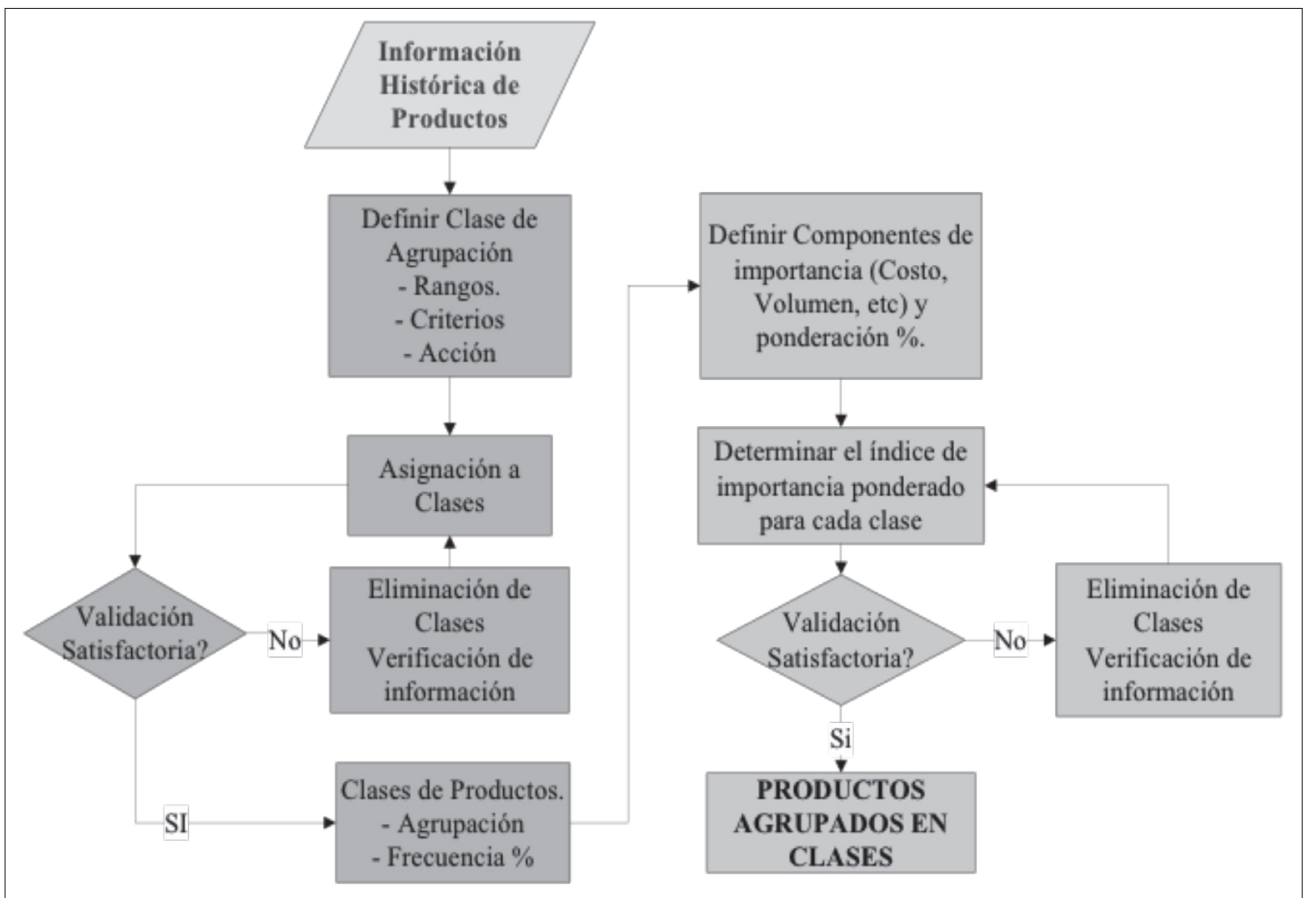

Figura 2. Metodología de clasificación de ítems

Fuente: elaboración propia.

Estos valores representan el $10 \%$ de la clase para el tipo A y el 3\% de la clase para el tipo b. Para el método de pronóstico se utilizó la mejor opción, un aplicativo comercial y especializado. Para determinar qué ítems se seleccionaban de cada tipo y de cada clase, se utilizó el ranking del sistema de clasi- ficación de la sección anterior, es decir, se tomaron los elementos más importantes de cada categoría según el criterio unificado de movimiento y de importancia, se le dio un código especial y requerido por el aplicativo. En la tabla 4 se relacionan las diferentes técnicas por cada clase de elementos.

Tabla 4. Comparación de técnicas por clase y el estimado

\begin{tabular}{|c|c|c|c|c|c|c|c|c|c|c|}
\hline Pronóstico & 1-A & 1-B & 2-A & 2-B & 3-A & 3-B & 4-A & 4-B & Real & EST. \\
\hline SE-EM & 6 & 8 & & & & & & & 318 & 214 \\
\hline SE-EA & 3 & 19 & & & & & & & 430 & 450 \\
\hline SE & 10 & 13 & 7 & 19 & 2 & 13 & 2 & 6 & 872 & 1695 \\
\hline SE-LA & & 2 & & & & & & & 82 & 46 \\
\hline SE-LM & 1 & 2 & & & & & & & 66 & 51 \\
\hline ML & & & & & & & & & 9 & \\
\hline DD & & & & & & & & & 25 & \\
\hline PMS & & & & & 1 & 3 & 1 & 2 & 46 & 180 \\
\hline TOTAL & 20 & 44 & 7 & 19 & 3 & 16 & 3 & 8 & 1848 & 2636 \\
\hline
\end{tabular}

Fuente: elaboración propia. 
De esta tabla se puede decir que las muestras marcan una tendencia a unificar la Suavización Exponencial (SE) con las Suavizaciones Exponenciales Aditivas (EA) y Multiplicativas (EM), y Lineal Aditiva (LA) y Lineal Multiplicativa (LM); por otro lado, técnicas de poca participación como los Modelos Lineales (ML) o los de datos discretos (DD) se pierden en el momento de hacer la proyección. Estas técnicas de suavización exponencial: aditivas y multiplicativas, son las que se enmarcan en el contexto de la suavización triple y de grado superior basadas en el mismo principio de volver a suavizar la componente base. Los modelos de suavización exponencial simple y doble pueden tratar con casi cualquier tipo de datos, siempre y cuando estos datos sean no estacionarios. Este método está basado en tres ecuaciones principales: una para el promedio, otra para la tendencia y otra para la estacionalidad. Dependiendo si la estacionalidad se modela de forma aditiva o multiplicativa.

Estos modelos son diferenciados para cada tipo de componente y reflejan cambios en las estructuras de las componentes de datos para cada elemento, razón por la cual no deberían simplificarse a otros modelos, como podrían ser los de suavización simple o los de promedios móviles. Se debe escoger una técnica de pronósticos, para ello se presentan las siguientes opciones:

1. Seleccionar una técnica para cada elemento del análisis, es decir, se debe construir 2637 modelos distintos con sus correspondientes parámetros de ejecución.

2. Seleccionar una técnica para cada clase y tipo con la selección de parámetros lo más generales posibles.

En general, se puede decir que con la primera opción se reducen los errores de los pronósticos pero se complica su cálculo, no solo por lo que representa su ejecución computacional, sino también por las implicaciones en las revisiones de los parámetros que se requieren en ciertas técnicas de pronósticos como los de suavización exponencial aditiva y multiplicativa. Con la segunda opción se aumentan los errores del pronóstico pero desde luego se simplifica considerablemente el cálculo de las estimaciones en términos de esfuerzo humano y computacional. Para este caso se escoge la opción dos, los pronósticos sugeridos se presentan en la tabla 5 de acuerdo con el análisis realizado a los datos del caso de aplicación, en la tabla 5 es la constante de suavización exponencial, $\mathrm{CV}$ es el coeficiente de variación como medida de variabilidad (Hopp y Spearman, 2001) cociente de la desviación sobre la media. d.l.c, $=$ de lo contrario y $\mathrm{k}$ es el número de datos para promedio móvil simple y doble.

Esta metodología se debe realizar para los productos en cada uno de los eslabones de la cadena, en este sentido, se considera oportuno descargar a las regionales del proceso de estimación de la demanda; en una muestra de SKU se determinó que los errores medidos en la desviación absoluta promedio o MAD (Mean Absolute Deviation) se aumentan cuando se decide realizar pronósticos independientes por ítem en cada central o regional, en este sentido, como el sistema de clasificación depende del número de ceros, cambia la política en cada una de estas regionales y varía su estimación. En la figura 3 se muestra el flujograma de proceso para aplicar esta metodología, integrándose con los resultados de la metodología de clasificación.

Tabla 5. Asignación de la técnica de pronóstico y sus parámetros a cada clase de ítem

\begin{tabular}{|c|c|c|}
\hline Clase & Técnica & Parámetros \\
\hline $\begin{array}{c}\text { Clase } 1 \\
-\mathrm{A}\end{array}$ & $\begin{array}{c}\text { Suavización expo- } \\
\text { nencial simple }\end{array}$ & $\begin{aligned} \mathrm{Si} C V<1 \rightarrow & =0,0001 ; \\
\mathrm{Si} C V<2 & \rightarrow=0,4 ; \\
\mathrm{SiCV}<3 \rightarrow & =0,0001 ; \\
\text { d.I.c. } & =0,7\end{aligned}$ \\
\hline $\begin{array}{c}\text { Clase } 1 \\
-B\end{array}$ & $\begin{array}{l}\text { Suavización expo- } \\
\text { nencial simple }\end{array}$ & $\begin{array}{c}\text { Si CV }<1 \rightarrow=0,3 ; \\
\text { Si CV }<2 \rightarrow=0,45 ; \\
\text { Si CV }<3 \rightarrow=0,01 ; \\
\quad \text { d.I.c. }=0,15\end{array}$ \\
\hline
\end{tabular}




\section{reflexión}

\begin{tabular}{|c|c|c|}
\hline Clase & Técnica & Parámetros \\
\hline $\begin{array}{l}\text { Clase } \\
2-\mathrm{A}\end{array}$ & $\begin{array}{l}\text { Suavización expo- } \\
\text { nencial simple }\end{array}$ & $=0,001$ \\
\hline $\begin{array}{l}\text { Clase } \\
2-B\end{array}$ & $\begin{array}{l}\text { Suavización expo- } \\
\text { nencial simple }\end{array}$ & $\begin{array}{c}\text { Si CV }<1 \rightarrow=0,00001 ; \\
\text { Si CV }<2 \rightarrow=0,0001 ; \\
\text { d.I.c. }=0,999\end{array}$ \\
\hline $\begin{array}{c}\text { Clase } 3 \\
-\mathrm{A}\end{array}$ & $\begin{array}{l}\text { Promedio móvil } \\
\text { doble }\end{array}$ & $\begin{array}{c}\text { Si CV }<2 \rightarrow k=2 ; \\
\text { Si CV }<3 \rightarrow k=7 \\
\text { d.l.c. } k=4\end{array}$ \\
\hline $\begin{array}{c}\text { Clase } 3 \\
-B\end{array}$ & $\begin{array}{l}\text { Promedio móvil } \\
\text { doble }\end{array}$ & $\begin{array}{c}\text { Si CV }<2 \rightarrow k=7 ; \\
\text { Si CV }<4 \rightarrow k=5 ; \\
\quad \text { d.I.c. } k=3\end{array}$ \\
\hline
\end{tabular}

\begin{tabular}{|c|c|c|}
\hline Clase & Técnica & Parámetros \\
\hline $\begin{array}{c}\text { Clase 4 } \\
-\mathrm{A}\end{array}$ & $\begin{array}{c}\text { Promedio móvil } \\
\text { doble }\end{array}$ & $\mathrm{k}=3$ \\
\hline $\begin{array}{c}\text { Clase 4 } \\
-\mathrm{B}\end{array}$ & $\begin{array}{c}\text { Promedio móvil } \\
\text { doble }\end{array}$ & $\begin{array}{c}\mathrm{Si} \mathrm{CV}<1 \rightarrow \mathrm{k}=3 ; \\
\text { Si CV }<3 \rightarrow \mathrm{k}=2 ; \\
\text { d.I.c. } \mathrm{k}=5\end{array}$ \\
\hline $\begin{array}{c}\text { Clase 5 - } \\
\text { A y B }\end{array}$ & Promedio móvil & Si CV $<1 \rightarrow \mathrm{k}=9 ;$ \\
& doble & Si CV $<3 \rightarrow \mathrm{k}=4 ;$ \\
& & d.I.c. $\mathrm{k}=2$
\end{tabular}

Fuente: elaboración propia.

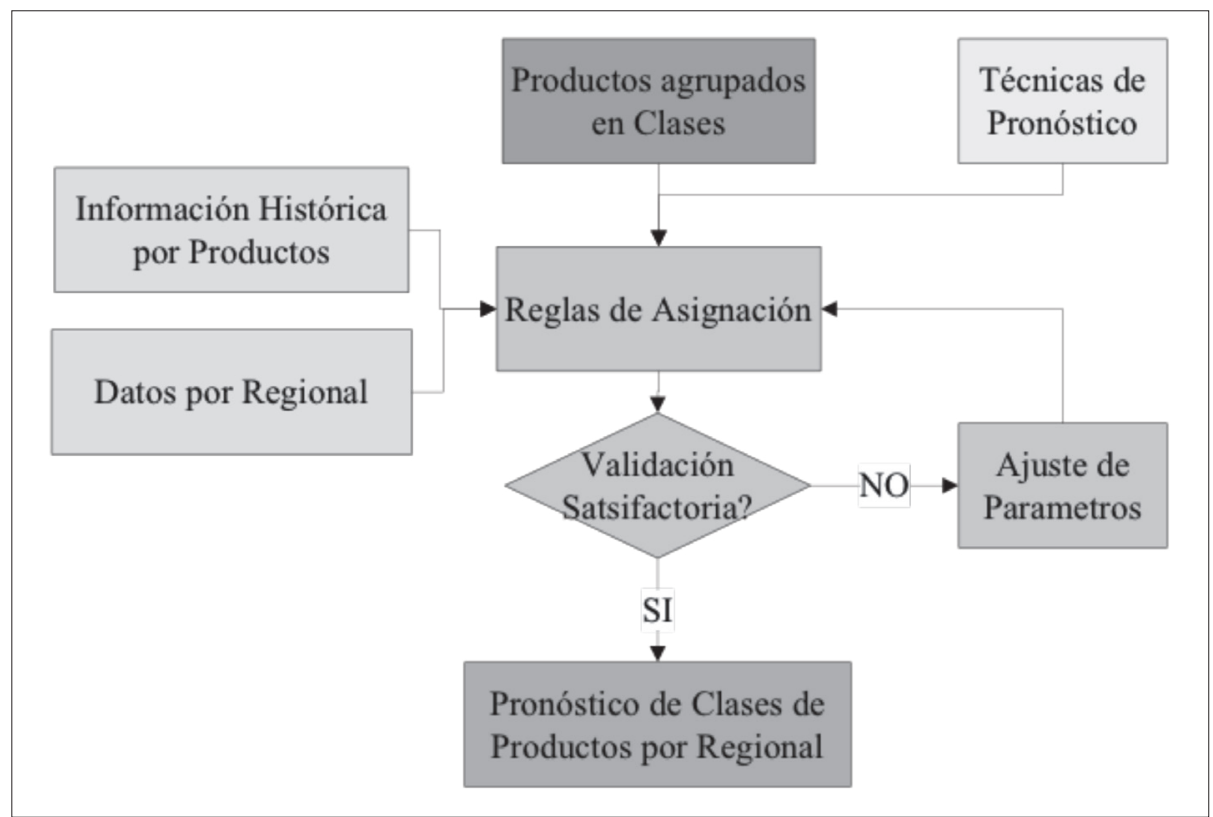

Figura 3. Metodología estimación de demanda de items y clases

Fuente: elaboración propia.

Por otro lado, se mitigan los efectos de permanencia, tendencia y estacionalidad al considerar por separado los valores de la demanda, razón muy importante a la hora de hacer las estimaciones. Sin embargo se considera importante que las regionales clasifiquen los pedidos de la siguiente manera:

- Pedidos ordinarios: son aquellos que se enmarcan dentro del pronóstico suministrado por logística o el encargado de hacerlos. En este sentido, las regionales pueden hacer observaciones de las estimaciones a futuro, o bien porque consideran que no se pueden ejecutar o bien porque se consideran menores a la información que disponen.

- Pedidos especiales: son aquellos que trascienden las expectativas del pronóstico, pueden 
incluir ítems sin estimaciones o en realidad nuevos productos. También pueden obedecer a demandas coyunturales que no ameritan ser consideradas en las demandas ya que salen como punto extremo.

En síntesis, se puede decir que para establecer el proceso de estimación de la demanda se hará una estimación por SKU y luego se "reparte" por regional y por ítem según los valores históricos en los que se ha basado su estimación.

\section{Resultados}

Este proyecto se implementó durante seis meses en la empresa caso de estudio en paralelo junto con la metodología anterior con el fin de comparar los resultados. Para evidenciar las mejoras del proceso de integrar las técnicas de pronósticos y las políticas de inventario, se puede decir que en el primer caso se hicieron pruebas como las que se muestran en la tabla 6 .

Tabla 6. Ejemplos de comparación por SKU. Metodología tradicional vs propuesta

\begin{tabular}{|c|c|c|c|c|c|c|}
\hline Mes & Código & Tipo & $\begin{array}{l}\text { Pronóstico } \\
\text { Tradicional }\end{array}$ & $\begin{array}{l}\text { Pronóstico } \\
\text { Propuesto }\end{array}$ & $\begin{array}{c}\text { Desviación P. } \\
\text { Tradicional }\end{array}$ & $\begin{array}{c}\text { Desviación P. } \\
\text { Propuesto }\end{array}$ \\
\hline \multirow{5}{*}{ Julio } & T129845 & Tuberías & $91.151,8$ & $95.097,0$ & $40.545,6$ & $44.490,9$ \\
\hline & T58337 & Tuberías & $70.654,3$ & $30.054,9$ & $27.792,8$ & 7806,7 \\
\hline & T391407 & Tuberías & $77.305,2$ & $51.001,8$ & $56.642,4$ & $30.339,0$ \\
\hline & & & & Desviación & $41.660,3$ & $27.545,5$ \\
\hline & & & & Mejora & $33,9 \%$ & \\
\hline \multirow{5}{*}{ Agosto } & T129845 & Tuberías & $61.866,4$ & $81.753,1$ & $59.757,8$ & $79.644,5$ \\
\hline & T58337 & Tuberías & $58.951,3$ & $37.397,6$ & $35.850,0$ & $14.296,3$ \\
\hline & Т391407 & Tuberías & $23.950,2$ & $41.901,2$ & 4212,6 & $22.163,6$ \\
\hline & & & & Desviación & $33.273,5$ & $38.701,5$ \\
\hline & & & & Mejora & $-16,3 \%$ & \\
\hline \multirow{5}{*}{ Septiembre } & T129845 & Tuberías & $6.3564,1$ & $57.859,4$ & $10.849,3$ & 5144,6 \\
\hline & T58337 & Tuberías & $52.612,3$ & $33.109,7$ & $24.547,1$ & 5044,5 \\
\hline & Т391407 & Tuberías & $32.386,1$ & $35.251,7$ & 4090,4 & 6956,0 \\
\hline & & & & Desviación & $13.162,3$ & 5715,0 \\
\hline & & & & Mejora & $56,6 \%$ & \\
\hline
\end{tabular}

Fuente: elaboración propia.

Como se observa, algunos productos se predicen mejor y otros no, sin embargo cuando se realizó la valorización de las estimaciones se encuentra que, a medida que el proyecto se consolida y dada su característica dinámica, las mejoras medidas en MAD se dan en promedio para el último trimestre de validación en un 10,2\%, como lo muestran los resultados de la tabla 7 . 


\section{reflexión}

Tabla 7. Asignación de la técnica de pronóstico y sus parámetros a cada clase de ítem

\begin{tabular}{|c|c|c|c|}
\hline Mes & MAD-anterior & MAD-nueva & Mejora (\%) \\
\hline Julio & $2.633 .834,7$ & $2.612 .507,1$ & 0,8 \\
\hline Agosto & $2.680 .345,6$ & $2.237 .873,2$ & 16,5 \\
\hline Septiembre & $2.404 .336,9$ & $2.078 .521,4$ & 13,6 \\
\hline PROMEDIO & 2572839,1 & 2309633,9 & 10,2 \\
\hline
\end{tabular}

Fuente: elaboración propia.

Por otro lado, se muestra la reducción de las existencias de producto terminado y de materiales de- bido a la mejora en la estimación de la demanda con el método de pronóstico y la clasificación de inventario descritos en el apartado de Clasificación por movimientos. Se tenía un promedio de $5,176 \mathrm{t} /$ mes y se pasó a un valor de 4,923 t/mes, lo que significa un ahorro de $253 \mathrm{t} / \mathrm{mes}$ que es un valor aproximado al $5 \%$ en el primer trimestre de uso de la metodología. De esta manera, se evidencia la mejora en procesos en la disminución de manipulación de inventario, transporte de productos, entre otros aspectos operativos, y también el ahorro en costos que genera a partir de la consolidación del proceso. En la figura 4 se muestra comparativamente la evolución de las ventas, la producción y el inventario, en la cual se observa una tendencia decreciente del inventario a un punto de estabilización al igual que una nivelación de la producción.

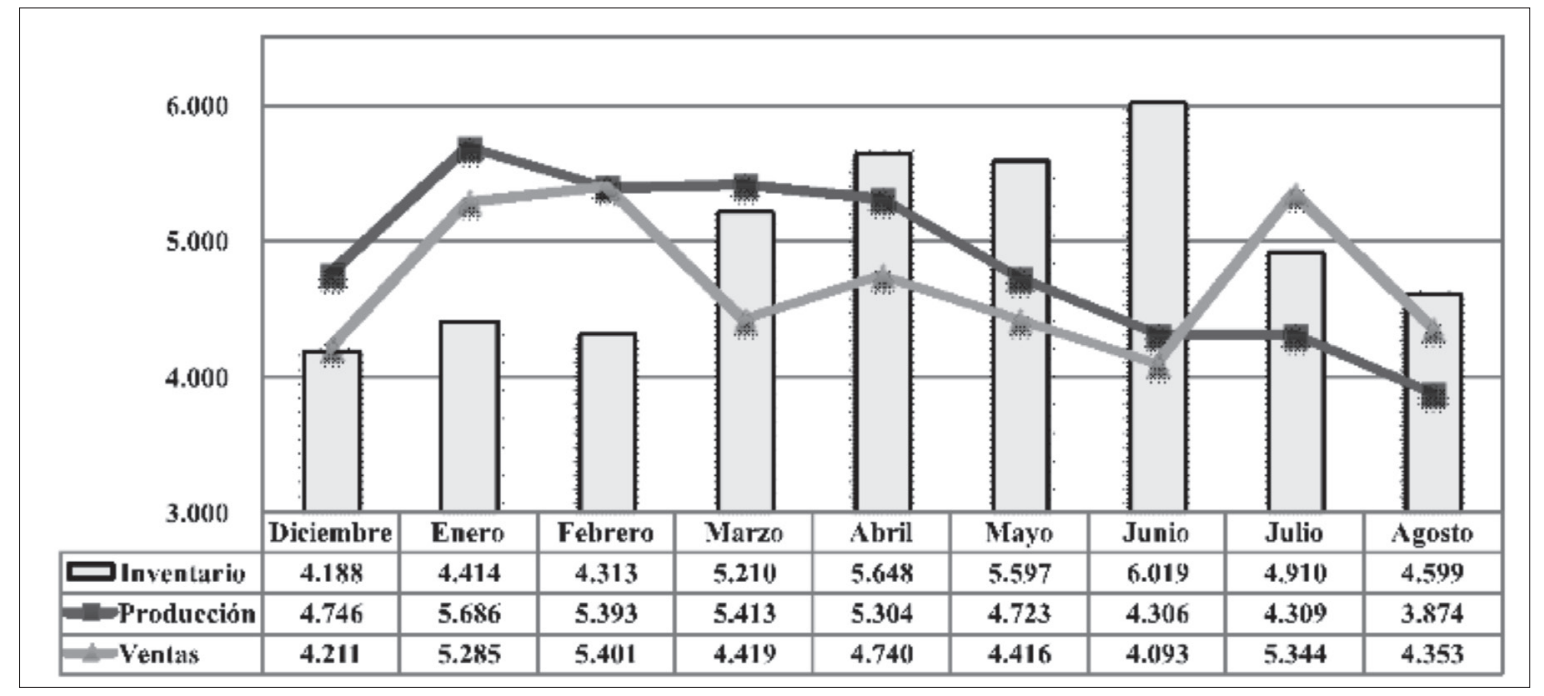

Figura 4. Comparativo ventas, producción e inventario (en toneladas)

Fuente: elaboración propia.

\section{CONCLUSIONES}

Los criterios para clasificar los ítems deben conciliar las características de rotación con los de valor, por lo tanto, se establecen los criterios de movimiento y los de importancia. Con el primero, se establece las clase y con el segundo el tipo. En el caso particular de la instancia, se encontró que existe un porcentaje significativo de ítems - con demanda cero en el horizonte de planeación o en el último año de dicho periodo, a los cuales se les debe analizar su baja o al menos establecer un pe- 


\section{reflexión}

ríodo de vigilancia para, en un próximo futuro, eliminar del maestro de materiales.

Los movimientos establecen seis categorías de las cuales solo se consideran cuatro con el fin de realizar los pronósticos. La clase 1 representa los ítems estables en el mercado -demanda en al menos el $87,5 \%$ de los períodos-. La clase 2 representa los elementos que están entrando o saliendo de la etapa de estabilidad - demanda entre el 50 y $85 \%$ de los períodos-. La clase 3 son los ítems que están en la fase de crecimiento, es decir aquellos que no tienen demanda más allá del $50 \%$ de los períodos pero esta preferencialmente se presenta en los últimos períodos del análisis. La clase 4 son para los ítems que se ubican en la etapa de declive, es decir, demandas en menos del $50 \%$ de los períodos, principalmente en los periodos iníciales del análisis. La clase 5 es para ítems sin movimiento en el último año y deben entrar en cuarentena o en periodo de observación. La clase 6 para elementos sin demanda, para los cuales se sugiere su baja.

El criterio de importancia se basa en múltiples atributos, en este caso son el costo, el peso y el volumen, si estos atributos son altos, el ítem debe estar en una clasificación más rigurosa, es decir, se debe ejercer mayor control sobre ellos. Para este caso se definieron dos tipos A y B. Tipo A son aquellos en donde la contribución marginal ponderada y acumulada es menor al $95 \%$. Tipo B para aquellos ítems con una contribución marginal ponderada mayor al $95 \%$.

Los pronósticos son específicos para cada ítem, pero esto conlleva a un manejo complejo del día a día, entonces dependiendo de la clasificación se decide establecer un modelo específico para ellos. De esto se tienen ocho modelos distintos que corresponde a las cuatro clases y los dos tipos. Las técnicas empleadas son la de suavización exponencial doble, suavización exponencial simple, promedio móvil doble y promedio móvil simple, con diferentes parámetros dependiendo del tipo. Para atender el sistema de estimación de la demanda en la cadena logística se sugiere hacer una sola estimación total y aplicar un factor por cada ítem para cada una de las centrales, esto reduce el margen de error en la estimación y disminuye los niveles de existencias.

\section{TRABAJOS FUTUROS}

Como trabajo futuro se plantea desarrollar una metodología integral de gestión de inventarios que considere la clasificación dinámica de los productos, la estimación de la demanda y la asignación de políticas de inventario para múltiples productos y eslabones en una cadena de suministro, la cual puede ayudar a los gestores de dichas cadenas a mitigar las variaciones de los inventarios y el nivel de servicio con sus clientes.

\section{REFERENCIAS}

Arora, K. (2005). Comprehensive production and operation management. Bangalore: Laxmi Publications.

Askin, R. G. \& Goldberg, J. B. (2002). Design and analysis of lean production systems. New York: Wiley.
Ballou, R. H. (2004). Business logistics/supply chain management: planning, organizing, and controlling the supply chain. Upper Saddle River, N.J.: Prentice Hall.

Borisov, V. N., Bulanov, A. A., Orlova, T. G. \& Kondratov, D. I. (2008). Forecast- 


\section{reflexión}

ing engineering manufacture operations with a view to their innovative development. Studies on Russian Economic Development, 19(4), 350-357. doi:10.1134/ S1075700708040047

Chen, J.-X. (2011). Peer-estimation for multiple criteria $\mathrm{ABC}$ inventory classification. Computers \& Operations Research, 38(12), 1784-1791. doi:10.1016/j.cor.2011.02.015

Chu, C.-W., Liang, G.-S., \& Liao, C.-T. (2008). Controlling inventory by combining $\mathrm{ABC}$ analysis and fuzzy classification. Computers \& Industrial Engineering, 55(4), 841851. doi:10.1016/j.cie.2008.03.006

Hadi-Vencheh, A. (2010). An improvement to multiple criteria $\mathrm{ABC}$ inventory classification. European Journal of Operational Research, 201 (3), 962-965. doi:10.1016/j. ejor.2009.04.013

Heizer, J. H. \& Render, B. (2010). Principles of Operations Management. Boston: Pearson Education.

Hopp, W. J. \& Spearman, M. L. (2008). Factory Physics: Foundations of Manufacturing Management. Irwin/McGraw-Hill.

Mendez Giraldo, G. A. (2003). Gerencia de manufactura Función de planeación. (Primera edición). Bogotá: Universidad Distrital Francisco José de Caldas.
Ng, W. L. (2007). A simple classifier for multiple criteria ABC analysis. European Journal of Operational Research, 177 (1), 344-353. doi:10.1016/j.ejor.2005.11.018

Partovi, F. Y. \& Anandarajan, M. (2002). Classifying inventory using an artificial neural network approach. Computers \& Industrial Engineering, 41 (4), 389-404. doi:10.1016/ S0360-8352(01)00064-X

Ramanathan, R. (2006). ABC inventory classification with multiple-criteria using weighted linear optimization. Computers \& Operations Research, 33(3), 695-700. doi:10.1016/j.cor.2004.07.014

Rezaei, J. \& Dowlatshahi, S. (2010). A rule-based multi-criteria approach to inventory classification. International Journal of Production Research, 48(23), 7107-7126. doi:10.1080/00207540903348361

Torabi, S. A., Hatefi, S. M. \& Saleck Pay, B. (2012). ABC inventory classification in the presence of both quantitative and qualitative criteria. Computers \& Industrial Engineering, 63(2), 530-537. doi:10.1016/j. cie.2012.04.011

Tsai, C.-Y, \& Yeh, S.-W. (2008). A multiple objective particle swarm optimization approach for inventory classification. International Journal of Production Economics, 114(2), 656-666. doi:10.1016/j.ijpe.2008.02.017 